\title{
Space-Time-Frequency Coded for Multiband-OFDM Based on IEEE 802.15.3a WPAN
}

\author{
Kamal MOHAMED-POUR, Hossein EBRAHIMI \\ Department of Electrical Engineering, K. N. Toosi University of Technology, Tehran, Iran \\ Email:kmpour@kntu.ac.ir \\ Received September 9, 2008; revised February 23, 2009; accepted April 15, 2009
}

\begin{abstract}
In this paper, Multiband-OFDM UWB system based on IEEE 802.15.3a standard is studied and simulated with spatial, time and frequency (STF)coding scheme. The using of STF coding method can guarantee both full symbol rate and full diversity advantages. The simulation results show that the STF code uses multipath-rich and random-clustering characteristics of UWB channel environment on the performance of MBOFDM system.
\end{abstract}

Keywords: IEEE 802.15.3a, UWB, MB-OFDM, MIMO, Space-Time-Frequency Coding

\section{Introduction}

Ultra wideband (UWB) systems are the first nomination for future wireless personal area networks (WPANs). The enormous band with availability provides the potential for very high data rates. The ultra wide bandwidth of UWB enables various WPAN applications such as highspeed wireless universal serial bus (WUSB) connectivity for personal computers and their accessories, high-quality real-time video and audio transmission, and cable replacement for home entertainment systems.

Currently, the multi-band orthogonal frequency division multiplexing (MB-OFDM) [1] is an important candidate for the physical layer within IEEE 802.15.3a standard.

On the other hand, the rich scattering multipath channel in UWB indoor environment provides an ideal transmission scenario for multiple antenna configurations. In this paper, we use a space-time-frequency coding (STFC) method [2] that can guarantee both full symbol rate and full diversity for performance improvement of MB-OFDM UWB systems over CM 1-CM 4 environment with 2ISO and 2I2O MIMO configurations.

The paper is organized as follows: In Section 2, an overview of STFC MB-OFDM UWB system is given. Section 3 gives the simulation results, and finally Section 4 concludes the paper.

\section{STFC MB-OFDM UWB System}

\subsection{UWB Channel Model}

The channel model is based on Saleh-Valenzuela model [3] according to IEEE 802.15.3a standard. The channel impulse response can be expressed as

$$
h_{i}(t)=X_{i} \sum_{l=0}^{L_{c}-1} \sum_{k=0}^{K_{c}-1} \alpha_{k, l}^{i} \delta\left(t-T_{l}^{i}-\tau_{k, l}^{i}\right)
$$

where $i$ represents the realization of the $i$-th impulse response, $\alpha_{k, l}^{i}$ is the multipath gain coefficients, $T_{l}^{i}$ is the delay of the $l$-th cluster, $\tau_{k, l}^{i}$ is the delay of $k$-th ray, and $X_{i}$ represents the log-normal shadowing. The cluster arrivals and the path arrivals within each cluster are modeled by Poisson processes

$$
\begin{aligned}
& p\left(T_{l} \mid T_{l-1}\right)=\Lambda \exp \left[-\Lambda\left(T_{l}-T_{l-1}\right)\right], \quad l>0 \\
& p\left(\tau_{k, l} \mid \tau_{(k-1), l}\right)=\lambda \exp \left[-\lambda\left(\tau_{k, l}-\tau_{(k-1), l}\right)\right], \quad k>0
\end{aligned}
$$

where $\Lambda$ and $\lambda$ (where $\lambda>\Lambda$ ) are the cluster arrival rate and ray arrival rate, respectively. Four set of channel model (CM) parameters for different measurement environments were defined, namely $\mathrm{CM} 1, \mathrm{CM} 2, \mathrm{CM} 3$, and $\mathrm{CM} 4$. Table 1 provides the model parameters of $\mathrm{CM}$ $1-\mathrm{CM} 4$ [4].

\subsection{STFC MB-OFDM Structure}

We consider a UWB multiband OFDM system with fast band-hopping rate that signal is transmitted on a frequency-band during one OFDM symbol interval, and then 
Table 1. The IEEE UWB channel parameters.

\begin{tabular}{ccccc}
\hline Parameters & CM 1 & CM 2 & CM 3 & CM 4 \\
\hline Condition & LOS & NLOS & NLOS & NLOS \\
$\Lambda(1 / \mathrm{nsec})$ & $0-4 \mathrm{~m}$ & $0-4 \mathrm{~m}$ & $4-10 \mathrm{~m}$ & $4-10 \mathrm{~m}$ \\
$\lambda(1 / \mathrm{nsec})$ & 2.5 & 0.5 & 2.1 & 2.1 \\
cluster decay factor & 7.1 & 5.5 & 14 & 24 \\
ray decay factor & 4.3 & 6.7 & 7.9 & 12 \\
$N_{\text {path }}(10 \mathrm{~dB})$ & 12.5 & 15.3 & 24.9 & 41.2 \\
$N_{\text {path }}(85 \%)$ & 20.8 & 33.9 & 64.7 & 123.3 \\
\hline
\end{tabular}

moved to a different frequency-band at the next interval. In Table 2 you can see the simulation parameters of MB-OFDM UWB system. The data is encoded by STF code words across $M_{t}$ transmit antennas, $N$ OFDM subcarriers, and K OFDM blocks. We suppose a frequencyselective fading channels based on S-V model, between any pair of transmit and receive antennas. Figure 1 represents the structure of system. Because of small wavelength in UWB environment and fast frequency hopping, consideration of independency between MIMO channel elements is reasonable. In this case, according to [2,5] the maximum achievable diversity is at most min $\left\{M_{t} M_{r}, \operatorname{rank}\left(R_{T}\right), K N M_{r}\right\}$, where $\mathrm{L}$ is the number of delay path and $R_{T}$ is the temporal correlation matrix.

We use repetition coded STF code [2] that is a full diversity code as follows:

$$
D_{S T F}=\mathbf{1}_{K \times 1} \otimes D_{S F}
$$

where $1_{K \times 1}$ is an all one matrix, $\otimes$ is tensor product, and $D_{S F}$ is a full diversity SF code of size $N \times M_{t}$ which have been proposed in [6]. At the transmitter, the information is jointly encoded across $M_{t}$ transmit antennas, M OFDM subcarriers, and K OFDM blocks. Each STF codeword is a $K N \times M_{t}$ matrix that can be expressed as a

$$
\boldsymbol{D}_{k}=\left[\begin{array}{lllll}
\boldsymbol{G}_{k, 1}^{T} & \boldsymbol{G}_{k, 2}^{T} & \ldots & \boldsymbol{G}_{k, P}^{T} & \mathbf{0}_{\left(N-P \Upsilon M_{t}\right) \times M_{t}}^{T}
\end{array}\right]
$$

where $P=\left\lfloor N / \Upsilon M_{t}\right\rfloor$ and $\Upsilon$ is an integer smaller than $\mathrm{N}$, which determines the number of jointly encoded subcarriers. Also

$$
\boldsymbol{G}_{k, P}=\left(\boldsymbol{I}_{K M_{t}} \otimes 1_{\Upsilon \times 1}\right)\left(\begin{array}{cc}
x_{p, 1} & x_{p, 2} \\
-x_{p, 2}^{*} & x_{p, 1}^{*}
\end{array}\right)
$$

where $x_{p, k} s$ are selected from QPSK or BPSK constellations. As mentioned earlier, we use repetition STFC which is based on Alamouti's structure. After STFC encoder, we add some preambles and headers for channel estimation and frame and packet synchronization. The baseband OFDM signal to be transmitted by $i$-th transmit antenna at the k-th OFDM block can be expressed as [7]

$$
x_{i}^{k}(t)=\sqrt{\frac{E}{M_{t}}} \sum_{n=0}^{N-1} d_{i}^{k}(n) \exp \left\{(j 2 \pi n \Delta f)\left(t-T_{C P}\right)\right\}
$$

where $d_{i}^{k}(n)$ represents the complex symbol to be transmitted over $n$-th subcarrier by $\mathrm{i}$-th transmit antenna during the k-th OFDM symbol period. Finally, after filtering, up conversion and band hopping, the trans-mitted signal over $\mathrm{i}$-th antenna is

$$
s_{i}(t)=\sum_{k=0}^{K-1} \operatorname{Re}\left\{x_{i}^{k}\left(t-k T_{S Y M}\right) \exp \left(j 2 \pi f_{c}^{k} t\right)\right\}
$$

In the receiver, after frequency dehopping, down converting and filtering, we have received signal at in matrix form as [2]

$$
\boldsymbol{Y}=\sqrt{\frac{E}{M_{t}}} \boldsymbol{D H}+\boldsymbol{Z}
$$

where $\boldsymbol{D}$ is the STF coded data, $\boldsymbol{H}$ is the MIMO channel matrix, and $\boldsymbol{Z}$ is complex baseband noise. Because of channel estimation pilots, we can determine $\boldsymbol{H}$, so we have

$$
\boldsymbol{W} \times \boldsymbol{Y}=\sqrt{\frac{E}{M_{t}}} \boldsymbol{W D H}+\boldsymbol{W Z}=\sqrt{\frac{E}{M_{t}}} \boldsymbol{D}+\boldsymbol{W Z}
$$

where $\boldsymbol{W}=\left(\boldsymbol{H}^{H} \boldsymbol{H}\right)^{-1} \boldsymbol{H}^{H}$. The receiver exploits a maximum likelihood detector over received signal matrix.

$$
\widehat{\boldsymbol{D}}=\arg \min \left\{\left\|\boldsymbol{Y}-\sqrt{\frac{E}{M_{t}}} \mathbf{D H}\right\|^{2}\right\}
$$

Therefore, the error probability will be as

$$
\left.P_{e}\right|_{\boldsymbol{H}}=Q\left(\sqrt{\frac{\rho}{2 M_{t}} \sum_{j=1}^{M_{r}}\|(\mathbf{D}-\widehat{\mathbf{D}}) \boldsymbol{H}\|^{2}}\right)
$$

Table 2. The MB-OFDM UWB parameters.

\begin{tabular}{cc}
\hline Information rate & $200 \mathrm{Mbps}$ \\
Number of Subcarriers & 128 \\
Channel coding & $5 / 8$ rate convolutional \\
Constellation & QPSK/BPSK \\
Data tones & 100 \\
$T_{F F T}$ & $242.4 \mathrm{nsec}$ \\
$T_{C P}$ & $60.6 \mathrm{nsec}$ \\
$T_{G I}$ & $9.5 \mathrm{nsec}$ \\
$T_{S Y M}$ & $312.5 \mathrm{nsec}$ \\
Decoder & Hard viterbi \\
\hline
\end{tabular}




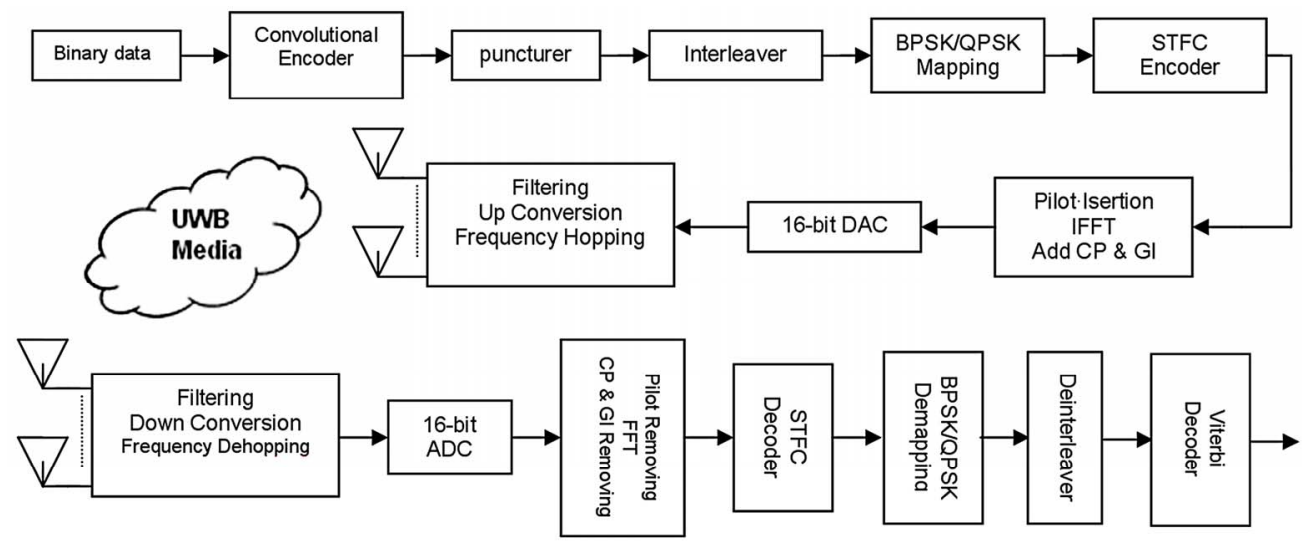

Figure 1. STFC MB-OFDM UWB system.

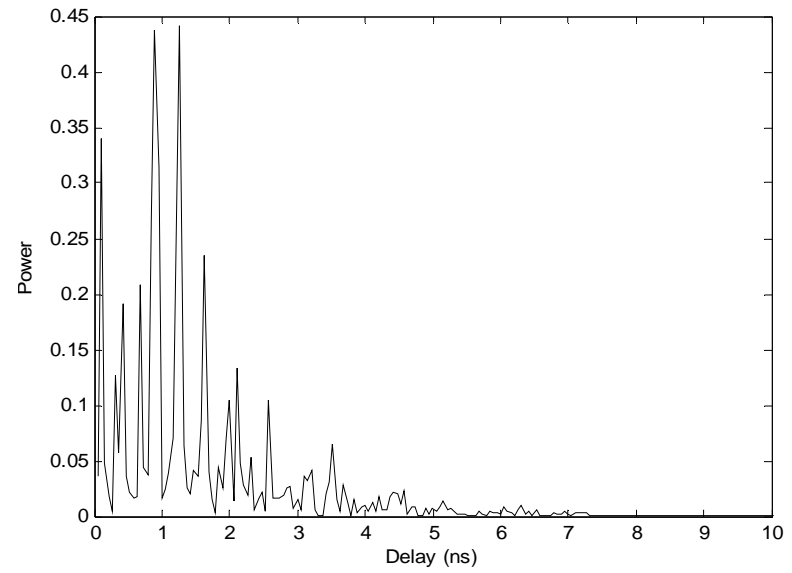

(a)

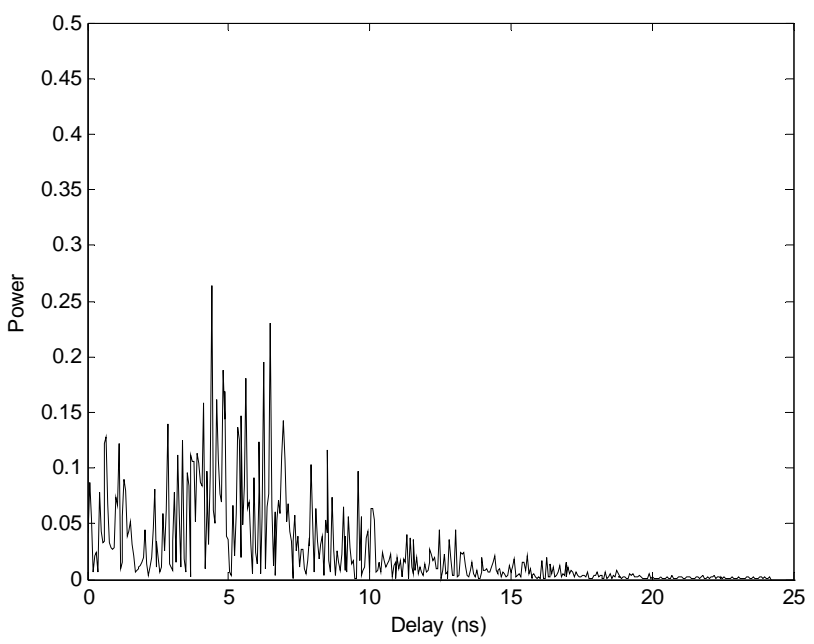

(c)

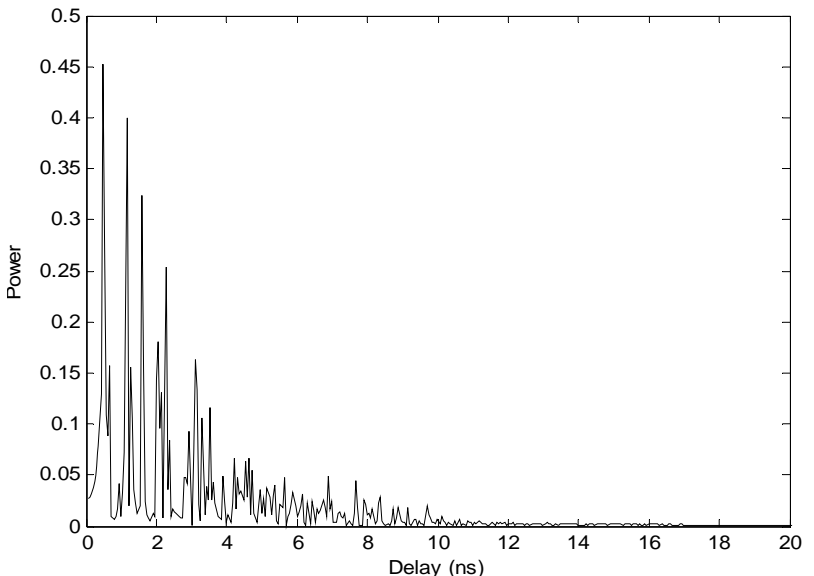

(b)

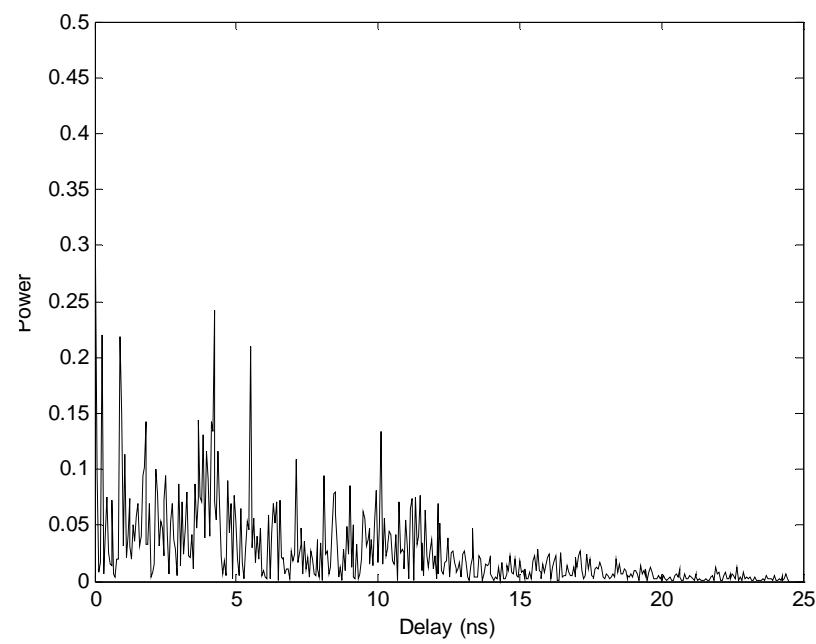

(d)

Figure 2. Simulated channel response; a) CM 1, b) CM 2, c) CM 3, d) CM 4.

\section{Simulation Results}

We performed simulations for a multiband UWB system with $N=128$ subcarriers and the subband bandwidth of
$528 \mathrm{MHz}$. Each OFDM symbol was of duration 242.42 $n s$. After adding the cyclic prefix of length $60.61 \mathrm{~ns}$ and the guard interval of length $9.47 \mathrm{~ns}$, the symbol duration became 312.5 ns. Figure 2 gives the simulated channel 
impulse responses for CM 1-CM 4. We used a repetition STFC based on Alamouti's structure that can guarantee full diversity [2]. Also we used 5/8-rate convolutional coding for improving performance. So our pure data rate was $200 \mathrm{Mbps}$. We first simulated UWB channel based on IEEE 802.15.3a standard. CM 2 is $0-4 \mathrm{~m}$, non line of sight channel, so it is reasonable to consider CM 2 for realistic application. Figure 3 gives the BER performance of MB-OFDM UWB as a function of SNR for CM 2 channel model, as frame length is 4200 QPSK symbols. In each frame, 600 symbols were preamble pilot for channel estimation. 100 channel realizations of IEEE 802.15.3a channel model (CM 1, 2, 3 and 4) were considered for the transmission of each symbol.

Figure 4 gives the BER performance of STFC coded MB-OFDM UWB as a function of SNR for CM 2 channel model without channel coding with the data jointly encoded across two subcarriers. The simulation results show that for CM 2 scenario, when $\mathrm{K}=1$ the $2 \mathrm{ISO}$ and $2 \mathrm{I} 2 \mathrm{O}$ configurations are almost $8.5 \mathrm{~dB}$ and $16 \mathrm{~dB}$ better than MB-OFDM, respectively. For $\mathrm{K}=2$, the $2 \mathrm{ISO}$ and $2 \mathrm{I} 2 \mathrm{O}$ configurations are almost $11.5 \mathrm{~dB}$ and $17.5 \mathrm{~dB}$ better than MB-OFDM, respectively.

Figure 5 gives the BER performance of STFC coded MB-OFDM UWB as a function of SNR for CM 2 and CM 4 for 2 ISO and $2 \mathrm{I} 2 \mathrm{O}$ configurations with the data jointly encoded across two subcarriers, when $\mathrm{K}=1$. In conventional MB-OFDM, performance for $\mathrm{CM} 4$ is worse than other scenarios, but it can be seen that the simulated performance for CM 4 is better than CM 2, when repetition STFC is employed. In coded system under CM 4 the coding gain is larger. It seems that space time frequency coding yields the MB-OFDM system can gain the multipath clustering property of UWB environments. In fact, when repetition STFC is employed, in comparison with other scenarios, CM 4 has the minimum correlation among OFDM subcarriers.

\section{Conclusions}

In this paper, MIMO-MB-OFDM has been studied. The simulation results indicate that the 2I2O STFC-MBOFDM scheme for UWB system shows much better performance compared with un-coded MB-OFDM. On the other hand, the performance of STF coded system can be improved by increasing the number of antenna, regardless of the random clustering behavior of UWB channels.

\section{References}

[1] A. Batra, et al., "Multi-band OFDM physical layer proposal for IEEE 802.15 task group 3a," IEEE 802.1503/268r3, Texas Instruments, March 2004.

[2] W. Su, Z. Safar, and K. J. R. Liu, "Towards maximum

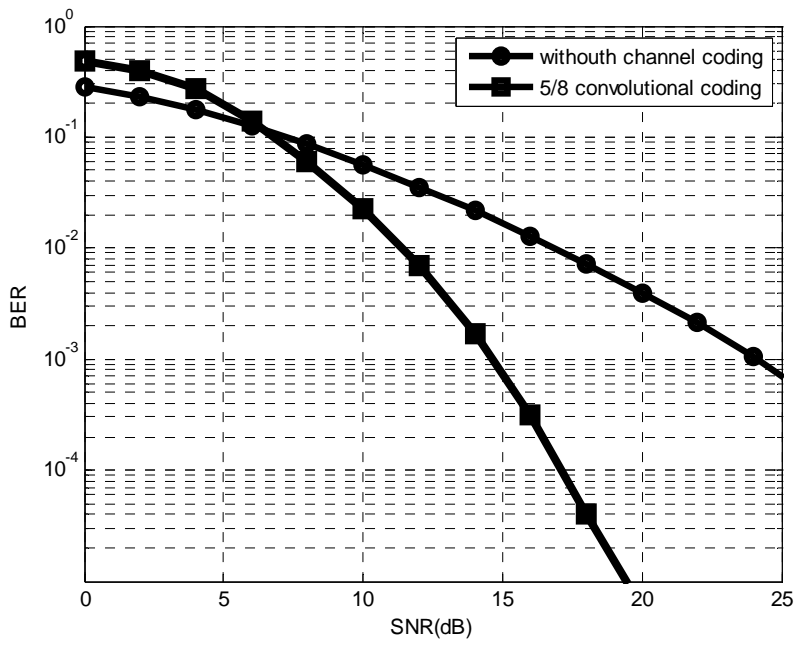

Figure 3. The performance of MB-OFDM UWB.

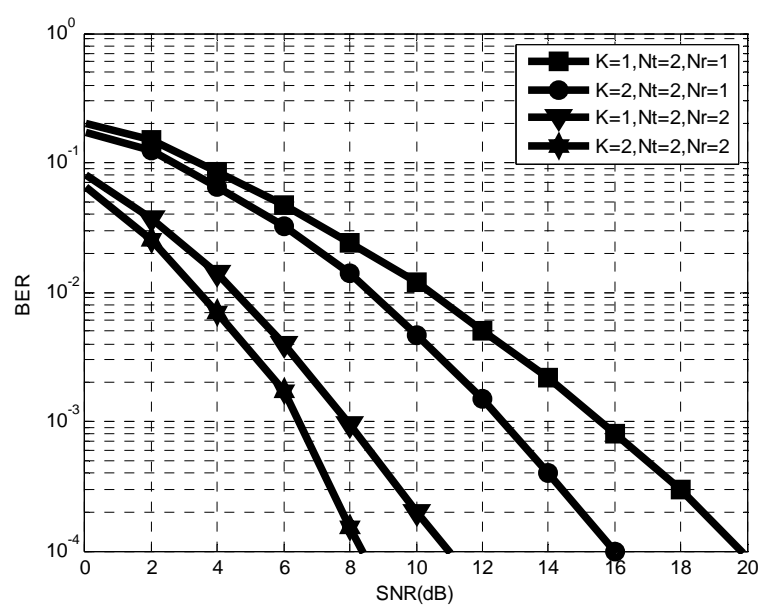

Figure 4. Performance of MB-OFDM for different MIMO configurations.

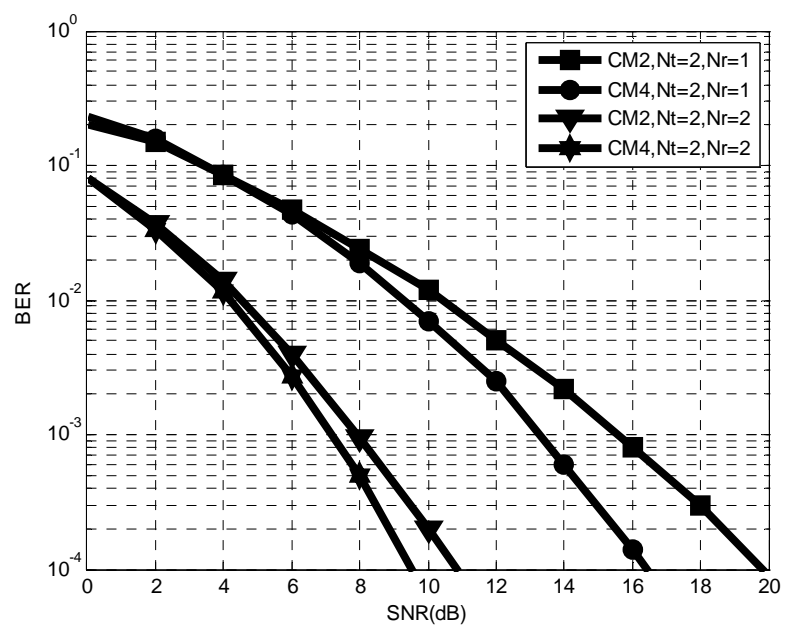

Figure 5. Performance comparison between CM 2 and CM 4.

achievable diversity in space, time and frequency: Per- 
formance analysis and code design," IEEE Trans. on Wireless Commun., Vol. 4, No. 4, pp. 1847-1857, Jul. 2005.

[3] IEEE 802.15WPAN High Rate Alternative PHY Task Group 3a (TG3a). Internet: www.ieee802.org/15/pub/ TG3a.html

[4] M. Ghavami, L. B. Michael, and R. Kohno, "Ultra wideband signals and systems in communication engineering," John Wiley \& Sons, Ltd, 2004.

[5] B. Lu, X. Wang, and K. R. Narayanan, "LDPC-based space-time coded OFDM systems over correlated fading channels: Performance analysis and receiver design," IEEE Trans. Commun., Vol. 50, No. 1, pp. 74-88, Jan. 2002.

[6] W. Su, Z. Safar, M. Olfat, and K. J. R. Liu, "Obtaining full-diversity space-frequency codes from space-time codes via mapping," IEEE Trans. Signal Processing, Vol. 51, pp. 2905-2916, Nov. 2003.

[7] W. P. Siriwongpairat, et al, " Multiband-OFDM MIMO coding framework for UWB communication systems," IEEE Trans. Signal Processing, Vol. 54, No. 1, Jan. 2006. 